\title{
Development and Validation of Single Nucleotide Polymorphism (SNP) Markers from an Expressed Sequence Tag (EST) Database in Olive Flounder (Paralichthys olivaceus)
}

\author{
Jung Eun Kim, Young Mee Lee, 'Jeong-Ho Lee, Jae Koo Noh, Hyun Chul Kim, Choul-Ji Park, \\ Jong-Won Park and Kyung-Kil Kim \\ Genetics and Breeding Research Center, NFRDI, Geoje 656-842, Korea
}

\begin{abstract}
To successful molecular breeding, identification and functional characterization of breeding related genes and development of molecular breeding techniques using DNA markers are essential. Although the development of a useful marker is difficult in the aspect of time, cost and effort, many markers are being developed to be used in molecular breeding and developed markers have been used in many fields. Single nucleotide polymorphisms (SNPs) markers were widely used for genomic research and breeding, but has hardly been validated for screening functional genes in olive flounder. We identified single nucleotide polymorphisms (SNPs) from expressed sequence tag (EST) database in olive flounder; out of a total 4,327 ESTs, 693 contigs and 514 SNPs were detected in total EST, and these substitutions include 297 transitions and 217 transversions. As a result, 144 SNP markers were developed on the basis of 514 SNP to selection of useful gene region, and then applied to each of eight wild and culture olive flounder (total 16 samples). In our experimental result, only 32 markers had detected polymorphism in sample, also identified 21 transitions and 11 transversions, whereas indel was not detected in polymorphic SNPs. Heterozygosity of wild and cultured olive flounder using the 32 SNP markers is 0.34 and 0.29 , respectively. In conclusion, we identified SNP and polymorphism in olive flounder using newly designed marker, it supports that developed markers are suitable for SNP detection and diversity analysis in olive flounder. The outcome of this study can be basic data for researches for immunity gene and characteristic with SNP.

Key words : Olive flounder, Paralichthys olivaceus, Single nucleotide polymorphisms (SNPs), Expressed sequence tag (EST), Heterozygosity
\end{abstract}

\section{INTRODUCTION}

The olive flounder (Paralichthys olivaceus) is an economically important fish in Korea and Japan, and total yields of aquacultured olive flounder increased and reached to 40,805 tons in 2011 (FAO) in Korea. Recently, breeding research is focused only culture techniques and develop- ment of research also insufficient to further increase production. Higher breeding research including the development of molecular markers is essential to produce of good breeds and high value fisheries and to enhance our competitiveness in fisheries industry. Identification and functional characterization of economic traits (or breeding traits) related genes, the development of molecular breeding

\footnotetext{
Manuscript received 28 November 2014, Received in revised form 5 December 2014, Accepted 8 December 2014

${ }^{\dagger}$ Corresponding Author : Jeong-Ho Lee, Genetics and Breeding Research Center, NFRDI, Geoje 656-842, Korea. Tel : +82-55-639-5811, Fax : +82-55639-5809, E-mail : jhlee7124@korea.kr

This is an Open Access article distributed under the terms of the Creative Commons Attribution Non-Commercial License (http:// creativecommons.org/licenses/by-nc/3.0) which permits unrestricted non-commercial use, distribution, and reproduction in any medium, provided the original work is properly cited.
} 
techniques using DNA markers to molecular breeding is meaningful (Hubert et al., 2009; Yoon, 2006). Moreover, application of these genes in the aquaculture (molecular selective breeding) has attracted worldwide attention.

Recently, large-scale DNA sequencing such as BAC library projects and EST projects have provided usable data for a great variety of species (Hayes et al., 2005). In particular, the sequence analysis was significantly increased in economic worth of fish. This information can be used to increase the accuracy of selection breeding research for economical traits, thereby increasing the rate of genetic gain and production efficiency.

The molecular marker is an important element for breeding research, and there are various methods to used for the research. The kind of molecular techniques are RAPD (random-amplified polymorphic DNA), RFLP (restriction fragment length polymorphism), AFLP (amplified fragment length polymorphism), MS (microsatellites) and SNP (single nucleotide polymorphisms) (Liu \& Cordes, 2004). Among possible molecular markers, microsatellites have been most useful for population genotyping technology, but is not suitable to high-quality data about influence gene expression and the functions. Unlike MS, single nucleotide polymerphisms (SNPs) are the most abundant type of variation in DNA sequence among individuals in a population (Fahrenkrug et al., 2002). Moreover, these markers appear to have low mutation rates (a mutation occurs every $2 \times 10^{8}$ nucleotides) and hence are extremely stable than other genetic markers (The International SNP Map Working Group, 2001). Although, SNPs have been recently proposed as an alternative to microsatellites for genotyping individuals (Vignal et al., 2002), have a disadvantage of time and cost-consuming when a certain selection mechanism does not exist. Whereas, if developed SNPs from of expressed sequence tags (ESTs) can be particularly valuable, also it is possible to reduce the time and cost for developing a useful SNP marker. In aquaculture species, SNPs are especially important if they cause differences in economic traits, or are linked to the mutations that do so.

For some aquaculture species, a large number of SNPs was developed from expressed sequence tag (EST) database as below; catfish (He et al., 2003), salmon (Hayes et al., 2007; Campbell \& Narum, 2011; Everett et al., 2011), sea bream (Hayes et al., 2007; Campbell \& Narum, 2011; Everett et al., 2011), Atlantic cod (Hubert, 2009), and turbot (Vera et al., 2011). But, SNP marker was hardly developed and validated in olive flounder even economically important fish species in Korea (He et al., 2011; He et al., 2012).

Wild and cultured olive flounder have different growth rate and resistance to disease because of differences in the growth environment. For example, wild olive flounder has generally small size and slow growth but strong immunity, whereas cultured have generally rapid growth and heavy weight but weak immunity. Each feature is associated with a SNP, and therefore SNP analysis using wild and culture samples can be a help to find regions involved superior traits. In this study, we develop useful marker to detect the single nucleotide polymorphism (SNP) from EST in olive flounder and then apply to sample of wild and cultured. Moreover, we were identified substitution of SNP and population diversity analysis using developed marker.

\section{MATERIALS AND METHODS}

\section{EST database and candidate SNP detection}

\section{1) ESTs database assemble}

In this study used polymorphic sites were identified from expressed sequence tags (ESTs) derived from $P$. olivaceus. Using to the previous studied EST data, bioinformatic analysis was conducted to determine gene identities using Gene Master Software (Ensoltek). ESTs were assembled in clusters of contiguous sequences (contig) using ICAtools program (Parsons, 1995). Gene annotation procedures and homology searches of the sequenced ESTs have been locally done by BLASTX for amino acid 
similarity comparisons. Matches with the Expect value (E) less than 10xe-4 were considered to be significant. All ESTs that were not identified as orthologs of known genes were designated as unknown EST clones.
2) Candidate SNP detection

Total of 755 SNP genes were selected from chromosome 24. The selection of SNP gene criteria is as below; the frequency of minor allele is 30 percent, each gene is related

Table 1. Sequences of primers used for the PCR

\begin{tabular}{|c|c|c|c|}
\hline Marker name & Forward primer & Reverse primer & Amplicon size \\
\hline PO_02 & CCTTCCAAGTGCTTCATGAGG & ACTGACAGTGTTGTCACTACC & 150 \\
\hline PO_03 & GCTGGTGACAGTGATGGTGA & ATAGGAGTTCGCAGTTCCGT & 400 \\
\hline PO_23 & ATCTGTCAACGCGAACAGTG & CTCATTCAGGAGGAAGCAGC & 180 \\
\hline PO_25 & TCACTGTTGGGCAAGACTCC & AАCCTCACCCCCTGTTCAAG & 150 \\
\hline PO_32 & ACGAGAACTGGGTTGTGTCT & TGTAGGAGCTGTAGTTGGGGT & 300 \\
\hline PO_34 & TTCATCCTCAGCTTCTCCCT & TGTCGTTCAGCTTCGAGGAGT & 200 \\
\hline PO_36 & GCACAACAAACGGTTAGCTAG & GACTCACCGCTGATCACTCT & 200 \\
\hline PO_44 & AAGTGCAAAGCTGTTCCAGA & TCCACTAAATGTCTCCAGGAG & 200 \\
\hline PO_46 & CAGATCAGGAAGACCTCCTACG & TCTCAATGTCCTTGCCAACG & 200 \\
\hline PO_47 & GGTTGTTCTGTATTGCGGCAC & GCTAGACAGAGAACTGCGAG & 150 \\
\hline PO_50 & GCATTAGTGCGCCACAAACT & CGCACTTGGTGCTTTTCACT & 200 \\
\hline PO_52 & АСТТССССТСССАСААСТСТ & GACATGCTCAGGTTCCTTGT & 200 \\
\hline PO_57 & TCTTGATAGTGCTGCCCCAC & CCTGAACTCTTACGTCCAGC & 300 \\
\hline PO_59 & GCAGGAGTTCTGACTGTGAC & ССТTCCAACСТCCATGATGG & 320 \\
\hline PO_61 & AGGAGTGTGCCTCAGGATGA & CTCAGACATGCTGTCCTGGA & 300 \\
\hline PO_62 & AGGCATAAGAACCCGGGAAG & GTGTATGTGTTAACCTGTCCCT & 200 \\
\hline PO_67 & TATTCCAGATGCTTGGCCAC & CTCTGTTGATCTCAGCTGCA & 200 \\
\hline PO_79 & CAGCGCATGTTTGAGAGTCT & TGCAGATATCCCTCCCACAG & 600 \\
\hline PO_86 & AAACTCTCCTTGCTGCCTTC & AAAGTTGACGGTAACGGAGG & 200 \\
\hline PO_91 & TGTCTCCATCCTTCATCAGCT & GGTACCATCGACTTCGAGGA & 300 \\
\hline PO_92 & GCTCTACACACAATAGAACCCA & CAAACCTGACGTCCAGAAGA & 200 \\
\hline PO_109 & GGGAGCAAACTCCTCTACGA & ATGTCACGTGCTAAAGGAGC & 200 \\
\hline PO_110 & TGAAGCATGTGAGAGCTTCG & AGGGACATAAGTCCATCGTCA & 300 \\
\hline PO_116 & CAGCTCTTTGAAAATGCCTGG & GGTATCTGACGTTCTCGTGG & 400 \\
\hline PO_121 & TGACTACCCCGATGCCTATG & GGACTTCTCGTTTAGCTGAGAATGC & 150 \\
\hline PO_122 & CTCCTTGAAGCCGATCATCG & ACCTAAAGCGGGGATTGCTC & 150 \\
\hline PO_126 & AGAGAAGCTCCACATCCACC & GGGTTCAGACCTTGCAGAGA & 200 \\
\hline PO_130 & CCTGAAATTAGACTCCATTCCTGG & AGTGTCTTTGAGTCGCAACG & 200 \\
\hline PO_136 & GCAGATGTACGGCATGTGTG & CAACAACTCCCATGCCAGA & 200 \\
\hline PO_172 & TGGACATCAAGACCAGACTGG & GAAGCTGTTGGTGGTGACGT & 250 \\
\hline PO_176 & ACTGGTTCTTGAAGAGCCTCC & CGGCTCTTGTGAAAGCAGA & 300 \\
\hline PO_179 & CTCATAGCGCAACATCACCA & CCGGTTGTTAGGTCCAAACCT & 250 \\
\hline
\end{tabular}


with immune and growth, and evenly exists on chromosome 24. As a result, 144 markers were selected, developed the primer for detecting of specific gene. Primers of SNPs were designed using Primer 3 software (Rozen and Skaletsky, 2000) adjusting length products to 100-300 bp (Table 1).

\section{Validation of SNP in olive flounder}

\section{1) Sampling, amplification and analysis}

Wild and cultured each of eight olive flounder were randomly obtained from East Sea and Jeju Island in Korea, respectively. Genomic DNA was extracted from pectoral fin with the standard method of phenol-chloroform (He et al., 2008) and a total of 144 primers were designed.

Amplification was carried out in the following conditions; in $20 \mathrm{uL}$ volume including 2 4 uL template DNA (100 $150 \mathrm{ng}$ ), $1 \times$ Gold Taq buffer (Amplified Biosystems), 2.5 $\mathrm{mM}$ of $\mathrm{MgCl}_{2}, 0.2 \mathrm{mM}$ of dNTP (Solgent), 10 pmol of both forward and reverse primers, 1.25 $\mathrm{U}$ of Gold taq DNA polymerase (Amplified biosystems) and $1 \mathrm{uL}$ dimethyl sulfoxide (Sigma). Thermal cycling was conducted on a Applied Biosystems (Model \#: 9902) as following conditions: initial denaturation at $95^{\circ} \mathrm{C}$ for $2 \mathrm{~min}, 35$ cycles of denaturation at $95^{\circ} \mathrm{C}$ for $30 \mathrm{~s}$, annealing temperature at $58^{\circ} \mathrm{C}$ for $30 \mathrm{~s}$, and extension at $72^{\circ} \mathrm{C}$ for $30 \mathrm{~s}$. Final extension step was performed at $72^{\circ} \mathrm{C}$ for $10 \mathrm{~min}$. Amplification of each SNP was checked on $2 \%$ agarose gel using electrophoresis. PCR product was purified using DNA purification Kit, sequenced in forward direction with the Big Dye termination reaction chemistry kit (Nucleogen), and analyzed with an ABI 3730xl Genetic Analyzer (Applied Biosystems).

Sequences were aligned with the CLUSTAL W algorithm using MEGA 5.05 software. Heterozygosity was estimated using Arlequin software 3.5 and polymorphic information content (PIC) value using Power Marker V.3.25 software.

\section{RESULTS}

\section{Identification of SNPs from EST database}

The olive flounder EST-derived database contains 4,327 ESTs and identified 693 contigs and 514 SNP were detected. The average of SNP frequency was 1 per $216 \mathrm{bp}$ of contig sequences. These substitutions included 297 transitions $(\mathrm{C} / \mathrm{T}, 155 ; \mathrm{A} / \mathrm{G}, 142)$ and 217 transversions $(\mathrm{A} / \mathrm{T}, 56 ; \mathrm{A} / \mathrm{C}$, 57; $\mathrm{T} / \mathrm{G}, 62 ; \mathrm{C} / \mathrm{G}, 42)$, with $\mathrm{C} / \mathrm{T}$ rate $(155,52.8 \%)$ has captured most of substitutions and $\mathrm{C} / \mathrm{G}$ being the least

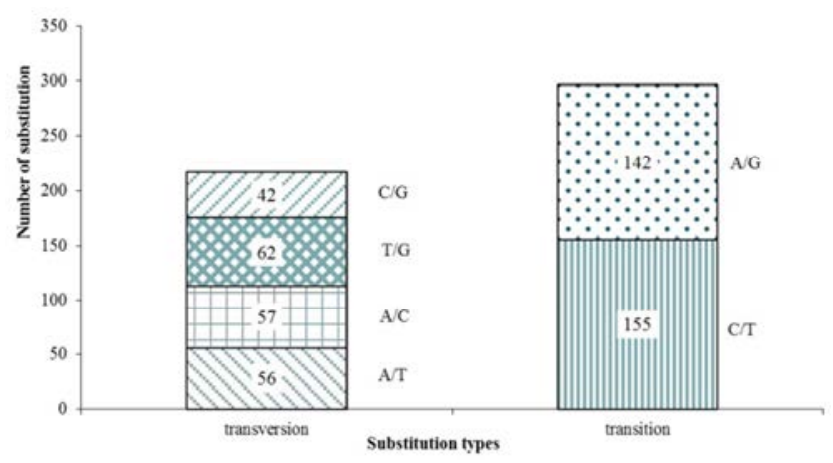

Fig. 1. Substitution types detected in the EST database of olive flounder.

Table 2. Summary statistics of SNP identification from olive flounder EST resources

\begin{tabular}{cc}
\hline \hline Parameter & EST resources SNPs \\
\hline Total number of ESTs searched & 4,327 \\
Number of contigs & 693 \\
Total SNPs detected & 514 \\
Total number of transitions & 297 \\
$\mathrm{C} / \mathrm{T}$ & 155 \\
$\mathrm{~A} / \mathrm{G}$ & 142 \\
$\mathrm{~A} / \mathrm{T}$ & 217 \\
$\mathrm{~A} / \mathrm{C}$ & 56 \\
$\mathrm{~T} / \mathrm{G}$ & 57 \\
$\mathrm{C} / \mathrm{G}$ & 62 \\
Total number of transversions & 42 \\
Total number of indels & 0 \\
Transition/transversion & 1.369 \\
\hline
\end{tabular}


common (42, 19.4\%) alteration observed (Fig. 1). A transition/ transversion ratio (ts/tv) was 1.369 , SNP substitutions result of development marker shows transitions rate higher than transversions rate (Table 2).

We were applied the designed primers on 16 sample (wild 8, cultured 8), and were performed PCR for amplifycation, with the result that 144 putative SNPs were selected

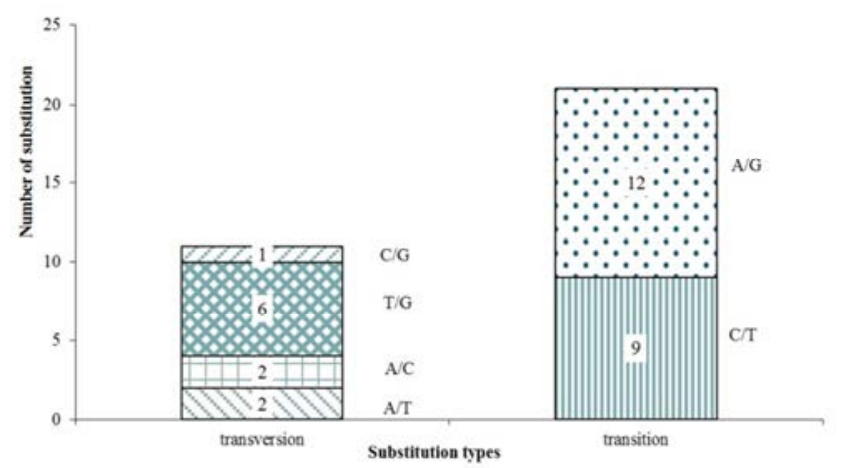

Fig. 2. Substitution types detected using the new designed marker in sample.

Table 3. Summary statistics of SNP identification from new designed marker

\begin{tabular}{cc}
\hline \hline Parameter & $\begin{array}{c}\text { Detected } \\
\text { SNPs }\end{array}$ \\
\hline Total designed primer pairs & 144 \\
Successful assays & 83 \\
Failed assays & 61 \\
Detected SNP substitutions from sample & 32 \\
Undetected SNP substitutions from sample & 51 \\
Total number of transitions & 21 \\
$\mathrm{C} / \mathrm{T}$ & 12 \\
$\mathrm{~A} / \mathrm{G}$ & 9 \\
$\mathrm{~A} / \mathrm{T}$ & 11 \\
$\mathrm{~A} / \mathrm{C}$ & 2 \\
$\mathrm{~T} / \mathrm{G}$ & 2 \\
$\mathrm{C} / \mathrm{G}$ & 6 \\
Total number of transversions & 1 \\
Transition/transversion & 0 \\
& 1.910 \\
\hline
\end{tabular}

for validation. Out of the 144 primer pairs, 25 primer did not amplify any product $(17.4 \%)$ and 36 primer failed to sequencing $(25.0 \%)$. Cause of failed sequencing is nonspecific bands $(8,5.6 \%)$, low PCR expression $(15,10.4 \%)$ and low alignment efficiency $(13,9.0 \%)$. But 83 markers $(57.6 \%)$ were successfully analyzed in sequencing analysis 51 out of 83 were confirmed monomorphic SNPs from the sample (wild 8, cultured 8), only 32 markers had detected polymorphic. 32 polymorphism SNP were observed in the variety genes region involved in immunity and protein synthesis (Table 4). The SNPs polymorphic are shown a total of 21 transitions $(\mathrm{C} / \mathrm{T}$ and $\mathrm{A} / \mathrm{G}), 11$ transversions (A/T, A/C, T/G and $\mathrm{C} / \mathrm{G})$, and indel was not detected in polymorphic SNPs (Fig. 2). With $\mathrm{C} / \mathrm{T}$ being the commonest (12) and $\mathrm{C} / \mathrm{G}$ the least common (1) alteration observed. Transition/transversion ratio (ts/tv) was 1.910 , SNP substitutions result of development marker shows transitions rate higher than transversions rate (Table 3 ). These results correspond with the EST resource SNP's ones.

\section{SNP genotype and allele frequency}

The calculation on frequency of genotyping and allele of 32 SNP markers, there were differences in allele frequency between wild and cultured olive flounder. In particular, Primer PO_03 (Allele: TT, 100\%), Primer PO_25 (Allele: TT, 100\%), Primer PO_91 (Allele: GG, 100\%), Primer PO_116 (Allele: CC, 100\%) marker had 100 percent one allele type in wild sample while cultured sample had various types. Meanwhile, Primer PO_36 (Allele: TT, 100\%), Primer PO_50 (Allele: CC, 100\%), Primer PO_79 (Allele: CC, 100\%), Primer PO_122 (Allele: AA, 100\%), Primer PO_179 (Allele: AA, 100\%) had one allele type in cultured sample while had various alleles in wild sample. The analysis on heterozygosity $(\mathrm{He})$ by sample showed 0.34 in wild sample and 0.29 in cultured sample, respecttively. The range of heterozygosity by marker was identified from 0.12 to 0.50 . Primer PO_61, Primer PO_62, Primer 
Table 4. Characteristics of the 32 polymorphic SNPs validated from the olive flounder EST database in this study

\begin{tabular}{|c|c|c|c|}
\hline Primer name & EST No. & Gene identity & $E$ value \\
\hline PO_02 & 98 & Apolipoprotein AI precursor & $2.00 \mathrm{E}-92$ \\
\hline PO_03 & 38 & Parvalbumin & $4.00 \mathrm{E}-52$ \\
\hline PO_23 & 23 & Gelsolin, like 1 & $0.00 \mathrm{E}+00$ \\
\hline PO_25 & 20 & Ahsg protein & $2.00 \mathrm{E}-41$ \\
\hline PO_32 & 32 & Trypsinogen 2 & $1.00 \mathrm{E}-132$ \\
\hline PO_34 & 16 & MHC class IIb antigen & $1.00 \mathrm{E}-114$ \\
\hline PO_36 & 15 & Ferritin heavy subunit & $4.00 \mathrm{E}-93$ \\
\hline PO_44 & 13 & Transferrin & $1.00 \mathrm{E}-140$ \\
\hline PO_46 & 13 & V-Fos transformation effector & $1.00 \mathrm{E}-140$ \\
\hline PO_47 & 12 & Family-2 cystatin & $1.00 \mathrm{E}-27$ \\
\hline PO_50 & 6 & Testis enhanced gene transcript-like protein & $2.00 \mathrm{E}-78$ \\
\hline PO_52 & 11 & Elastase 2 precursor & $1.00 \mathrm{E}-148$ \\
\hline PO_57 & 10 & Elastase 1 precursor & $1.00 \mathrm{E}-139$ \\
\hline PO_59 & 9 & Alpha-2-HS-glycoprotein & $3.00 \mathrm{E}-60$ \\
\hline PO_61 & 9 & Apolipoprotein E1 & $5.00 \mathrm{E}-86$ \\
\hline PO_62 & 9 & Triosephosphateisomerase B & $1.00 \mathrm{E}-127$ \\
\hline PO_67 & 7 & Alpha-1-antiproteinase-like protein & $1.00 \mathrm{E}-129$ \\
\hline PO_79 & 6 & Glutathione-S-transferase & $1.00 \mathrm{E}-107$ \\
\hline PO_86 & 5 & Ornithinedecarboxylaseantizyme large isoform & $2.00 \mathrm{E}-86$ \\
\hline PO_91 & 5 & Troponin $\mathrm{C}$, fast skeletal & $2.00 \mathrm{E}-71$ \\
\hline PO_92 & 4 & Natural killer enhancing factor & $1.00 \mathrm{E}-111$ \\
\hline PO_109 & 3 & Glyceraldehyde 3-phosphate dehydrogenase & $3.00 \mathrm{E}-60$ \\
\hline PO_110 & 3 & Pyruvate kinase & $4.00 \mathrm{E}-68$ \\
\hline PO_116 & 3 & Glycerol-3-phosphate dehydrogenase 1 (soluble) & $1.00 \mathrm{E}-118$ \\
\hline PO_121 & 69 & Cytochrome c oxidase subunit I & $0.00 \mathrm{E}+00$ \\
\hline PO_122 & 65 & Cytochrome c oxidase subunit II & $1.00 \mathrm{E}-105$ \\
\hline PO_126 & 7 & Lactate dehydrogenase-A; NAD+:lactate oxidoreductase; LDH-A & $1.00 \mathrm{E}-166$ \\
\hline PO_130 & 7 & Nephrosin & $1.00 \mathrm{E}-109$ \\
\hline PO_136 & 5 & Proteasome subunit $\mathrm{C} 10-11$ & $1.00 \mathrm{E}-110$ \\
\hline PO_172 & 2 & Ovel protein similar to type I cytokeratin,enveloping layer & $2.00 \mathrm{E}-71$ \\
\hline PO_176 & 2 & Ornithine decarboxylase antizyme small isoform & $2.00 \mathrm{E}-74$ \\
\hline PO_179 & 2 & NADH dehydrogenase subunit 2 & $2.00 \mathrm{E}-64$ \\
\hline
\end{tabular}

PO_121 marker had the highest value of Heat 0.50 while Primer PO_126 marker had the lowest value of 0.12 . The estimation of polymorphic information content (PIC) by marker ranged from 0.11 to 0.38 (Table 5).

\section{DISCUSSIONS}

Genomic regions of immune and protein synthesisrelated genes is directly related to life of organisms so that 
Table 5. Frequencies of genotype and allele for the SNPs in olive flounder

\begin{tabular}{|c|c|c|c|c|c|c|c|c|}
\hline \multirow{3}{*}{$\begin{array}{c}\text { SNP marker } \\
\text { Primer PO_02 }\end{array}$} & \multicolumn{5}{|c|}{ Frequencies (\%) } & \multirow{3}{*}{$\mathrm{He}$} & \multirow{3}{*}{$\begin{array}{c}\text { Total } \\
\mathrm{He}\end{array}$} & \multirow{3}{*}{ PIC } \\
\hline & \multicolumn{3}{|c|}{ SNP genotype } & \multicolumn{2}{|c|}{ Allele } & & & \\
\hline & $\mathrm{CC}$ & $\mathrm{CT}$ & $\mathrm{TT}$ & $\mathrm{C}$ & $\mathrm{T}$ & & & \\
\hline Wild & 50.00 & 37.50 & 12.50 & 68.75 & 31.25 & 0.43 & 0.45 & 0.35 \\
\hline Cultured & 50.00 & 25.00 & 25.00 & 62.50 & 37.50 & 0.47 & & \\
\hline Primer PO_03 & GG & $\mathrm{TG}$ & $\mathrm{TT}$ & G & $\mathrm{T}$ & & & \\
\hline Wild & 0.00 & 0.00 & 100.00 & 0.00 & 100.00 & 0.00 & 0.22 & 0.19 \\
\hline Cultured & 25.00 & 0.00 & 75.00 & 25.00 & 75.00 & 0.38 & & \\
\hline Primer PO_23 & AA & $\mathrm{AG}$ & GG & A & G & & & \\
\hline Wild & 37.50 & 50.00 & 12.50 & 62.50 & 37.50 & 0.47 & 0.42 & 0.33 \\
\hline Cultured & 57.14 & 42.86 & 0.00 & 68.75 & 18.75 & 0.34 & & \\
\hline Primer PO_25 & $\mathrm{CC}$ & $\mathrm{CT}$ & $\mathrm{TT}$ & $\mathrm{C}$ & $\mathrm{T}$ & & & \\
\hline Wild & 0.00 & 0.00 & 100.00 & 0.00 & 100.00 & 0.00 & 0.12 & 0.12 \\
\hline Cultured & 12.50 & 0.00 & 87.50 & 12.50 & 87.50 & 0.22 & & \\
\hline Primer PO_32 & AA & $\mathrm{AG}$ & GG & A & $\mathrm{G}$ & & & \\
\hline Wild & 50.00 & 37.50 & 12.50 & 68.75 & 31.25 & 0.43 & 0.34 & 0.28 \\
\hline Cultured & 75.00 & 25.00 & 0.00 & 87.50 & 12.50 & 0.22 & & \\
\hline Primer PO_34 & $\mathrm{CC}$ & $\mathrm{CT}$ & TT & $\mathrm{C}$ & $\mathrm{T}$ & & & \\
\hline Wild & 25.00 & 25.00 & 50.00 & 37.50 & 62.50 & 0.47 & 0.38 & 0.30 \\
\hline Cultured & 12.50 & 0.00 & 7.00 & 12.50 & 87.50 & 0.22 & & \\
\hline Primer PO_36 & GG & GT & $\mathrm{TT}$ & G & $\mathrm{T}$ & & & \\
\hline Wild & 0.00 & 37.50 & 62.50 & 18.75 & 81.25 & 0.30 & 0.17 & 0.16 \\
\hline Cultured & 0.00 & 0.00 & 100.00 & 0.00 & 100.00 & 0.00 & & \\
\hline Primer PO_44 & GG & GT & $\mathrm{TT}$ & G & $\mathrm{T}$ & & & \\
\hline Wild & 25.00 & 12.50 & 62.50 & 31.25 & 68.75 & 0.43 & 0.34 & 0.28 \\
\hline Cultured & 0.00 & 25.00 & 75.00 & 12.50 & 87.50 & 0.22 & & \\
\hline Primer PO_46 & $\mathrm{CC}$ & $\mathrm{CT}$ & $\mathrm{TT}$ & $\mathrm{C}$ & $\mathrm{T}$ & & & \\
\hline Wild & 37.50 & 62.50 & 0.00 & 68.75 & 31.25 & 0.43 & 0.45 & 0.35 \\
\hline Cultured & 50.00 & 25.00 & 25.00 & 62.50 & 37.50 & 0.47 & & \\
\hline Primer PO_47 & $\mathrm{AA}$ & $\mathrm{AT}$ & $\mathrm{TT}$ & A & $\mathrm{T}$ & & & \\
\hline Wild & 37.50 & 62.50 & 0.00 & 68.75 & 31.25 & 0.43 & 0.45 & 0.35 \\
\hline Cultured & 37.50 & 50.00 & 12.50 & 62.50 & 37.50 & 0.47 & & \\
\hline Primer PO_50 & $\mathrm{CC}$ & $\mathrm{CT}$ & $\mathrm{TT}$ & $\mathrm{C}$ & $\mathrm{T}$ & & & \\
\hline Wild & 50.00 & 12.50 & 37.50 & 56.25 & 43.75 & 0.49 & 0.34 & 0.28 \\
\hline Cultured & 100.00 & 0.00 & 0.00 & 100.00 & 0.00 & 0.00 & & \\
\hline PrimerPO_52 & AA & $\mathrm{AG}$ & GG & $\mathrm{A}$ & $\mathrm{G}$ & & & \\
\hline Wild & 0.00 & 37.50 & 62.50 & 18.75 & 81.25 & 0.30 & 0.38 & 0.30 \\
\hline Cultured & 12.50 & 37.50 & 50.00 & 31.25 & 68.75 & 0.43 & & \\
\hline Primer PO_57 & $\mathrm{AA}$ & $\mathrm{AG}$ & GG & A & $\mathrm{G}$ & & & \\
\hline Wild & 75.00 & 25.00 & 0.00 & 87.50 & 12.50 & 0.22 & 0.22 & 0.19 \\
\hline Cultured & 75.00 & 25.00 & 0.00 & 87.50 & 12.50 & 0.22 & & \\
\hline
\end{tabular}


Table 5. Continued

\begin{tabular}{|c|c|c|c|c|c|c|c|c|}
\hline \multirow{3}{*}{$\begin{array}{c}\text { SNP marker } \\
\text { Primer PO_59 }\end{array}$} & \multicolumn{5}{|c|}{ Frequencies (\%) } & \multirow{3}{*}{$\mathrm{He}$} & \multirow{3}{*}{$\begin{array}{c}\text { Total } \\
\mathrm{He}\end{array}$} & \multirow{3}{*}{ PIC } \\
\hline & \multicolumn{3}{|c|}{ SNP genotype } & \multicolumn{2}{|c|}{ Allele } & & & \\
\hline & $\mathrm{CC}$ & $\mathrm{CT}$ & TT & $\mathrm{C}$ & $\mathrm{T}$ & & & \\
\hline Wild & 12.50 & 75.00 & 12.50 & 50.00 & 50.00 & 0.50 & 0.47 & 0.36 \\
\hline Cultured & 62.50 & 25.00 & 12.50 & 75.00 & 25.00 & 0.38 & & \\
\hline PrimerPO_61 & AA & $\mathrm{AC}$ & $\mathrm{CC}$ & $\mathrm{A}$ & $\mathrm{C}$ & & & \\
\hline Wild & 37.50 & 62.50 & 0.00 & 68.75 & 31.25 & 0.43 & 0.50 & 0.38 \\
\hline Cultured & 12.50 & 37.50 & 50.00 & 31.25 & 68.75 & 0.43 & & \\
\hline PrimerPO_62 & $\mathrm{CC}$ & $\mathrm{CT}$ & $\mathrm{TT}$ & $\mathrm{C}$ & $\mathrm{T}$ & & & \\
\hline Wild & 12.50 & 62.50 & 25.00 & 43.75 & 56.25 & 0.49 & 0.50 & 0.38 \\
\hline Cultured & 25.00 & 62.50 & 12.50 & 56.25 & 43.75 & 0.49 & & \\
\hline PrimerPO_67 & $\mathrm{CC}$ & $\mathrm{CT}$ & TT & $\mathrm{C}$ & $\mathrm{T}$ & & & \\
\hline Wild & 50.00 & 0.00 & 50.00 & 50.00 & 50.00 & 0.50 & 0.42 & 0.33 \\
\hline Cultured & 83.33 & 0.00 & 16.67 & 83.33 & 16.67 & 0.28 & & \\
\hline Primer PO_79 & $\mathrm{CC}$ & $\mathrm{CT}$ & $\mathrm{TT}$ & $\mathrm{C}$ & $\mathrm{T}$ & & & \\
\hline Wild & 42.86 & 28.57 & 28.57 & 57.14 & 42.86 & 0.49 & 0.38 & 0.30 \\
\hline Cultured & 100.00 & 0.00 & 0.00 & 100.00 & 0.00 & 0.00 & & \\
\hline PrimerPO_86 & $\mathrm{AA}$ & AT & TT & A & $\mathrm{T}$ & & & \\
\hline Wild & 16.67 & 83.33 & 0.00 & 58.33 & 41.67 & 0.49 & 0.47 & 0.36 \\
\hline Cultured & 16.67 & 0.00 & 83.33 & 16.67 & 83.33 & 0.28 & & \\
\hline Primer PO_91 & $\mathrm{AA}$ & $\mathrm{AG}$ & GG & A & G & & & \\
\hline Wild & 0.00 & 0.00 & 100.00 & 0.00 & 100.00 & 0.00 & 0.23 & 0.20 \\
\hline Cultured & 28.57 & 0.00 & 71.43 & 28.57 & 71.43 & 0.41 & & \\
\hline Primer PO_92 & AA & $\mathrm{AG}$ & GG & A & $\mathrm{G}$ & & & \\
\hline Wild & 12.50 & 12.50 & 75.00 & 18.75 & 81.25 & 0.30 & 0.30 & 0.26 \\
\hline Cultured & 0.00 & 28.57 & 71.43 & 18.75 & 81.25 & 0.30 & & \\
\hline PrimerPO_109 & GG & GT & TT & G & $\mathrm{T}$ & & & \\
\hline Wild & 87.50 & 12.50 & 0.00 & 93.75 & 6.25 & 0.12 & 0.22 & 0.19 \\
\hline Cultured & 62.50 & 37.50 & 0.00 & 81.25 & 18.75 & 0.30 & & \\
\hline Primer PO_110 & $\mathrm{CC}$ & $\mathrm{CT}$ & TT & $\mathrm{C}$ & $\mathrm{T}$ & & & \\
\hline Wild & 0.00 & 62.50 & 37.50 & 31.25 & 68.75 & 0.43 & 0.40 & 0.32 \\
\hline Cultured & 12.50 & 25.00 & 62.50 & 25.00 & 75.00 & 0.38 & & \\
\hline PrimerPO_116 & $\mathrm{CC}$ & $\mathrm{CT}$ & TT & $\mathrm{C}$ & $\mathrm{T}$ & & & \\
\hline Wild & 100.00 & 0.00 & 0.00 & 100.00 & 0.00 & 0.00 & 0.38 & 0.30 \\
\hline Cultured & 50.00 & 25.00 & 25.00 & 62.50 & 37.50 & 0.47 & & \\
\hline PrimerPO_121 & AA & $\mathrm{AG}$ & GG & $\mathrm{A}$ & G & & & \\
\hline Wild & 12.50 & 0.00 & 87.50 & 12.50 & 87.50 & 0.22 & 0.50 & 0.38 \\
\hline Cultured & 87.50 & 0.00 & 12.50 & 87.50 & 12.50 & 0.22 & & \\
\hline Primer PO_122 & AA & $\mathrm{AG}$ & GG & A & G & & & \\
\hline Wild & 75.00 & 0.00 & 25.00 & 75.00 & 25.00 & 0.38 & 0.22 & 0.19 \\
\hline Cultured & 100.00 & 0.00 & 0.00 & 100.00 & 0.00 & 0.00 & & \\
\hline
\end{tabular}


Table 5. Continued

\begin{tabular}{|c|c|c|c|c|c|c|c|c|}
\hline \multirow{3}{*}{$\begin{array}{c}\text { SNP marker } \\
\text { Primer PO_126 }\end{array}$} & \multicolumn{5}{|c|}{ Frequencies (\%) } & \multirow{3}{*}{$\mathrm{He}$} & \multirow{3}{*}{$\begin{array}{c}\text { Total } \\
\mathrm{He}\end{array}$} & \multirow{3}{*}{ PIC } \\
\hline & \multicolumn{3}{|c|}{ SNP genotype } & \multicolumn{2}{|c|}{ Allele } & & & \\
\hline & AA & $\mathrm{AC}$ & $\mathrm{CC}$ & A & $\mathrm{C}$ & & & \\
\hline Wild & 0.00 & 12.50 & 87.50 & 6.25 & 93.75 & 0.12 & 0.12 & 0.11 \\
\hline Cultured & 0.00 & 12.50 & 87.50 & 6.25 & 93.75 & 0.12 & & \\
\hline Primer PO_130 & AA & $\mathrm{AG}$ & GG & A & G & & & \\
\hline Wild & 12.50 & 75.00 & 12.50 & 50.00 & 50.00 & 0.50 & 0.47 & 0.36 \\
\hline Cultured & 12.50 & 25.00 & 62.50 & 25.00 & 75.00 & 0.38 & & \\
\hline Primer PO_136 & $\mathrm{CC}$ & $\mathrm{CT}$ & $\mathrm{TT}$ & $\mathrm{C}$ & $\mathrm{T}$ & & & \\
\hline Wild & 62.50 & 25.00 & 12.50 & 75.00 & 25.00 & 0.38 & 0.38 & 0.30 \\
\hline Cultured & 50.00 & 50.00 & 0.00 & 75.00 & 25.00 & 0.38 & & \\
\hline Primer PO_172 & $\mathrm{CC}$ & $\mathrm{CG}$ & GG & $\mathrm{C}$ & G & & & \\
\hline Wild & 50.00 & 50.00 & 0.00 & 75.00 & 25.00 & 0.38 & 0.47 & 0.36 \\
\hline Cultured & 25.00 & 50.00 & 25.00 & 50.00 & 50.00 & 0.50 & & \\
\hline Primer PO_176 & $\mathrm{CC}$ & $\mathrm{CT}$ & TT & $\mathrm{C}$ & $\mathrm{T}$ & & & \\
\hline Wild & 12.50 & 62.50 & 25.00 & 43.75 & 56.25 & 0.49 & 0.43 & 0.34 \\
\hline Cultured & 0.00 & 37.50 & 62.50 & 18.75 & 81.25 & 0.30 & & \\
\hline Primer PO_179 & AA & $\mathrm{AG}$ & GG & A & $\mathrm{G}$ & & & \\
\hline Wild & 75.00 & 0.00 & 25.00 & 75.00 & 25.00 & 0.38 & 0.22 & 0.19 \\
\hline Cultured & 100.00 & 0.00 & 0.00 & 100.00 & 0.00 & 0.00 & & \\
\hline
\end{tabular}

He: expected heterozygosity; PIC: polymorphic information contents

its importance is well known (He et al., 2011; He et al., 2012). In addition, single base substitution of specific genomic region can be a positive or negative a factor for survival, growth and adaptation to the environment. Because these usefulness, the SNP research has been actively proceeding in various fish species; but hasn't yet been reported on olive flounder. In our experiments, discovery of SNP based on EST database in olive flounder and developed a valuable marker for applications to various genomic analyses. The SNPs polymorphism show that a total of 21 transitions $(\mathrm{C} / \mathrm{T}$ and $\mathrm{A} / \mathrm{G})$, and 11 transversions (A/T, A/C, T/G and $\mathrm{C} / \mathrm{G}$ ) while transitions are higher than transversions. This result is similar with other previous research (Vera et al., 2011). These result suggest that transitions are more easily substituted than transversion, because purine-purine (transitions) substitution is easy and stable than purine-pyrimidine (transversions) substitution.

Moreover, 32 SNPs genomic region of identified polymorphism are related to immune response and protein synthesis, functional changes in the these proteins causes the phenotypic change. The single nucleotide substitution of the specific region might influences on immunity and survival of organisms. In particular, glutathione S-transferase, nephrosin, elastases factors, MHC II alpha beta antigens and apolipoprotein involved in innate immunity (Dijkstra et al., 2013; Tsai ea al., 2004; Vera et al., 2011). In addition, ferritin has an important role for iron storage and detoxification, the storage of iron is closely related to the immune response to bacterial infection protected. Finally, transferrin could be related to the inflammation and enhanced resistance to oxidative stress by parasite infections (Paramá et al., 2007). Also, SNP detected genes in olive flounder show a 
high similarity with immune-related genes in mammals (Vera et al., 2011). The innate immune response is an important and highly developed defence mechanism against pathogens in fish. Examples of innate immunity include anatomic barriers, mechanical removal of pathogens, bacterial antagonism, pattern-recognition receptors, antigen-nonspecific defense compounds, complement pathways, phagocytosis, and inflammation fish (Ellis et al., 2001). The research outcome can be used as basis data for a study to identify the relationship between the nucleotide substitution in the immune-related genes specific region and immune response.

The allele frequency analysis using newly developed SNP marker shows that a variety of alleles is present in wild olive flounder samples, but does not shows the diversity in a few cultured samples. It shows the necessity to use different markers for the wild and cultured sample for genome analysis. Primer PO_36, Primer PO_50, Primer PO_79, Primer PO_122, Primer PO_179 marker are useful to detect SNP for wild olive flounder samples, while Primer PO_03, Primer PO_25, Primer PO_91, Primer PO_116 are suitable for cultured samples. In addition, this specific marker is important to distinguish between wild and culture olive flounder sample.

The diversity analysis using developed SNP marker in our research shows the heterozygosity value is higher in the wild sample of 0.34 than the cultured sample of 0.29 , meaning higher diversity of the wild than the cultured. The cultured sample has got smaller diversity due to continuous inbreeding in the group without any inflow of new species (new mother group), resulting in lower diversity than the wild (Xing et al., 2014). Result of our heterozygosity is a correlation with diversity study outcome of olive flounder using microsatellite (Jung et al., 2009). It supports the appropriateness and usefulness of newly developed SNP marker to apply not only to SNP detection but also to other diversity analysis.

In conclusion, we was identified the substitution using the new marker from sample, it supports that development marker on this study is suitable for SNP detection in olive flounder. In addition, the marker is an appropriate tool for diversity analysis. The outcome of this study will be used in the selection of breeding with economic traits and will facilitate the molecular breeding programs of olive flounder.

\section{ACKNOWLEDGEMENT}

This work was supported by a grant from the National Fisheries Research and Development Institute (NFRDI), contribution number RP-2014-BT-017.

\section{REFERENCES}

Campbell NR, Narum SR (2011) Development of 54 novel single-nucleotide polymorphism (SNP) assays for sockeye and coho salmon and assessment of available SNPs to differentiate stocks within the Columbia River. Mol Ecol Resour 11:20-30.

Cenadelli S, Maran V, Bongioni G, Fusetti L, Parma P, Aleandri R (2007) Identification of nuclear SNPs in gilthead seabream. J Fish Biol 70:399-405.

Dijkstra JM, Grimholt U, Leong J, Koop BF, Hashimoto K (2013) Comprehensive analysis of MHC class II genes in teleost fish genomes reveals dispensability of the peptide-loading DM system in a large part of vertebrates. BMC Evol Biol 260:26-13.

Ellis AE (2001) Innate host defence mechanisms of fish against viruses and bacteria. Dev Comp Immunol 25: 827-839.

Everett MV, Grau ED, Seeb JE (2011) Short reads and non-model species: exploring the complexities of next generation sequence assembly and SNP discovery in the absence of a reference genome. Mol Ecol Resour 11: 93-108.

Fahrenkrug SC, Freking BA, Smith TP, Rohrer GA, Keele 
JW (2002) Single nucleotide polymorphism (SNP) discovery in porcine expressed genes. Anim Genet 33: 186-195.

Hayes B, Laerdahl JK, Lien S, Moen T, Berg P, Hindar K, Davidson WS, Koop BF, Adzhubei A and Hoyheim B (2007) An extensive resource of single nucleotide polymorphism markers associated with Atlantic salmon (Salmo salar) expressed sequences. Aquaculture 265:8290.

Hayes B, Sonesson AK, Gjerde B (2005) Evaluation of three strategies using DNA markers for traceability in aquaculture species. Aquaculture 250:70-81.

He C, Chen L, Simmons M, Li P, Kim S, Liu ZJ (2003) Putative SNP discovery in interspecific hybrids of catfish by comparative EST analysis. Anim Genet 34: $445-448$

He F, HS Wen, SL Dong, B Shi, CF Chen (2008) Identification of single nucleotide polymorphism cytochrome P450-c19a and its relation to reproductive traits in Japanese flounder (Paralichthys olivaceus). Aquaculture 279:177-181.

He F, Ma RQ, Wen HS, Li JF, Mu WJ, Liu M, Zhang YQ, Hu J, Qun L (2012) Polymorphysims of CYP17-I gene in the exons were associated with the reproducetive endocrine of Japanese flounder (Paralichthys olivaceus). J Anim Sci 6:794-799.

He F, Wen HS, Li JF, Yu DH, Ma RQ, Shi D, Mu WJ, Zhang YQ, Hu J, Liu M, Han GW, Zhang JN, Wang QQ, Yuan UR, Liu Q (2011) Single nucleotide polymorphisms of the GnRHR gene associated with reproductive traits of Japanese flounder (Paralichthys olivaceus). J Anim Sci 4:463-470.

Hohenlohe PA, Amish SJ, Catchen JM, Allendorf FW, Luikart G (2011) Next-generation RAD sequencing identifies thousands of SNPs for assessing hybridization between rainbow and westslope cutthroat trout. Mol Ecol Resour 11:117-122.
Hubert S, Bussey JT, Higgins B, Curtis BA, Bowman S (2009) Development of single nucleotide polymerphism markers for Atlantic cod (Gadus morhua) using expressed sequences. Aquaculture 296:7-14.

Jung DS, Noh JG, Myung JI, Lee JH, Kim HC, Park CJ, Min BH, Ha DS, Jeoun CY (2009) Genetic variability comparison of wild populations and cultured stocks of flounder Paralichthys olivaceus based on microsatellited DNA Markers. Kor J Ichthyol 21:221-226.

Liu ZJ, Cordes JF (2004) DNA marker technologies and their applications in aquaculture genetics. Aquaculture 238:1-37.

Paramá A, Castro R, Arranz JA, Sanmartín ML, Lamas J, Leiro J (2007) Scuticociliate cysteine proteinases modulate turbot leucocyte functions. Fish Shellfish Immunol 23: 945-956.

Parsons JD (1995) Improved tools for DNA comparison and clustering. Comput Appl Biosci 11:603-613.

Rozen S, Skaletsky H (2000) Primer3 on the WWW for general users and for biologist programmers. Methods in Mol Biol 132:365-386.

The International SNP Map Working Group (2001) A map of human genome sequence variation containing 1.42 million single nucleotide polymorphisms. Nature 409: 928-933.

Tsai PL, Chen CH, Huang CJ, Chou CM, Chang GD (2004) Purification and cloning of an endogenous protein inhibitor of carp nephrosin, an astacin metalloproteinase. J Biol Chem 279:19-32.

Vera M, Antonio J, Dios A, Millán A, Pardo AG, Bouza C, Hermida M, Fernández C, Herrán , Molina-Luzón M, Paulino M (2011) Validation of single nucleotide polymorphism (SNP) markers from an immune Expressed Sequence Tag (EST) turbot, Scophthalmus maximus. Aquaculture 313:31-41.

Vignal A, Milan D, San Cristobal M, Eggen A (2002) A review on SNP and other types of molecular markers 
JE Kim, YM Lee, J-H Lee, JK Noh, HC Kim, C-J Park, J-W Park, K-K Kim

and their use in animal genetics. Genet Sel Evol 34: 275-305.

Xing K, Gao ML, Li HJ (2014) Genetic differentiation between natural and hatchery populations of Manila clam (Ruditapes philippinarum) based on microsatellite markers. Genet Mol Res 13:237-245.

Yoon JM (2006) Genetic differences and variations in freshwater crab (Eriocheir sinensis) and swimming crab (Portunus trituberculatus). Dev Repord 6:19-32. 